\title{
BMJ Open What are the important design features of personal health records to improve medication adherence for patients with long-term conditions? A systematic literature review
}

\author{
Elisavet Andrikopoulou, ${ }^{01,2}$ Philip Scott, ${ }^{1}$ Helena Herrera, ${ }^{3}$ Alice Good ${ }^{1}$
}

To cite: Andrikopoulou E, Scott P, Herrera $\mathrm{H}$, et al. What are the important design features of personal health records to improve medication adherence for patients with long-term conditions? A systematic literature review. BMJ Open 2019;9:e028628. doi:10.1136/ bmjopen-2018-028628

- Prepublication history and additional material for this paper are available online. To view these files, please visit the journal online (http://dx.doi. org/10.1136/bmjopen-2018028628).

Received 17 December 2018 Revised 02 July 2019 Accepted 23 August 2019

A) Check for updates

(C) Author(s) (or their employer(s)) 2019. Re-use permitted under CC BY-NC. No commercial re-use. See rights and permissions. Published by BMJ.

${ }^{1}$ School of Computing, Faculty of Technology, University of Portsmouth, Portsmouth, UK ${ }^{2}$ School of Computing, Buckingham Building, Lion Terrace, Portsmouth, UK ${ }^{3}$ School of Pharmacy and Biomedical Sciences, Faculty of Science, University of Portsmouth, Portsmouth, UK

Correspondence to Elisavet Andrikopoulou; elisavet.andrikopoulou@port. ac.uk

\section{ABSTRACT}

Objectives This systematic literature review aims to identify important design features of the electronic personal health record (PHR) that may improve medication adherence in the adult population with long-term conditions.

Data sources PubMed (including MEDLINE), CINAHL, Science Direct (including EMBASE), BioMed Central, ACM digital, Emerald Insight, Google Scholar and Research Gate.

Methods Studies that were published between 1 January 2002 and 31 May 2018 in English were included if the participants were adults, with at least one longterm condition, were able to self-administer their medication and were treated in primary care settings. The quality of evidence was assessed with the Grading of Recommendations Assessment, Development, and Evaluation (GRADE) system and the risk of bias was appraised using the Cochrane risk of bias tool.

Results From a total of 27 studies that matched the inclusion criteria, 12 were excluded due to low quality of evidence, 10 were rated moderate and 5 were rated high quality. All the included studies had low sample size and limited follow-up duration. Thirteen of the included studies found that the use of a PHR has increased medication adherence. The identified design features are reminders, education, personalisation and tailoring, feedback and alerts, gamification, medication management, medical appointment management, diary and self-monitoring, health condition management, set goals, patient's blog and tethered. It was impossible to draw conclusions as to which feature is important to what group of patients and why. The most frequently identified conditions were HIV and diabetes. This review did not identify any papers with negative results. It was not possible to numerically aggregate the PHR effect due to high heterogeneity of the medication adherence measurement, study type, participants and PHRs used.

Conclusion Although we found recurrent evidence that PHRs can improve medication adherence, there is little evidence to date to indicate which design features facilitate this process.

PROSPERO registration number CRD42017060542.
Strengths and limitations of this study

- This is a systematic literature review that follows the Preferred Reporting Items for Systematic Reviews and Meta-Analyses guidelines.

- The protocol was published before conducting the study to avoid data-driven decisions.

- A comprehensive literature search was carried out using eight major research paper databases, hand-searching and snowballing.

- All included studies were quality assessed using well-cited tools and documentation.

- A study limitation is that only studies that were published in English language were included.

\section{INTRODUCTION}

A 2018 healthcare consultant report calls for focusing on healthcare value instead of increasing the size of the current systems to accommodate for the rising demand. ${ }^{1}$ Healthcare expenditures were $16.5 \%$ of gross domestic product (GDP) in USA and $11.5 \%$ of GDP for Switzerland in 2016. ${ }^{1}$ NHS policy anticipates that increasing usage of health apps by patients will reduce demand on healthcare services. ${ }^{2}$ The NHS Long Term $\mathrm{Plan}^{3}$ policy document focuses on increasing personalisation in healthcare to improve quality of life and public health and aspires that information and communication technology (ICT) can reduce healthcare costs and improve healthcare outcomes over the next 5 years. This assertion, however, is not based on scientific research, so the claimed benefits remain aspirational.

Currently, $74 \%$ of UK nationals older than 45 years old and almost all younger adults less than 45 years old are using the internet nearly every day. ${ }^{4}$ Based on the results of a 2017 US survey, roughly $40 \%$ of people living with a long-term condition were interested in using 
technology to assist them with medication, diagnosis, test results and managing their condition in their home environment. ${ }^{5}$

A form of ICT that could potentially benefit the patients and the healthcare services is the electronic personal health record (PHR). A PHR has been defined as "online systems that include collections of patients' healthcare and medical data, which use health informatics standards to enable patients to share, organize and manage these data according to their own views". ${ }^{6}$ Some of the claimed benefits of PHRs are the ability of PHR to improve patient outcomes, decrease care cost, allow patients the ability to self-manage their health, increase access to care especially in remote areas, empower patients and improve medication adherence. $^{78}$

Medication adherence is a well-known challenge in healthcare ${ }^{910}$ and is related to a large number of factors such as side effects, ${ }^{11}$ forgetfulness ${ }^{9}$ or effective self-management ${ }^{12}$ and is affected by psychological factors and beliefs. ${ }^{12}$ Medication adherence can be defined as 'the extent to which a person's behaviour towards their medication intake, corresponds with agreed recommendations from a health care provider' ${ }^{10}$ The WHO reported that in developed countries the medication adherence in patients with long-term conditions averages to $50 \% .^{13}$ There is limited evidence whether PHRs actually improve medication adherence in chronically ill adults and no evidence synthesis as to which PHR design features are the most effective.

Polypharmacy refers to the simultaneous use of multiple medications and has been associated with several poor health outcomes including medication adherence. ${ }^{14-16}$ The effects of poor medication adherence are greater for people with polypharmacy. ${ }^{16}$ The effect is greater since the number of people with multiple long-term conditions is also rising. ${ }^{17}$ It is estimated that in UK, more than one-third of patients with at least one long-term illness do not adhere to their medication regime. ${ }^{18}$

A number of systems are currently in practice to use ICT in order to store, manage and employ health and medical information. These ICTs are developed by coders who are implementing human-computer interaction (HCI) principles. ${ }^{19}$ The HCI discipline is used to improve the usability of the software to developers, especially in healthcare settings. ${ }^{20}$ The NHS standards for PHRs ${ }^{21}$ provide guidance on good practice for their development in England, but they do not provide enough details or guidelines on what design features should a PHR include nor evidence on how these features impact health outcomes.

Although a number of strategies and interventions have been identified to assist patients' medication adherence, ${ }^{22}{ }^{23}$ the number of approaches related to PHRs is surprisingly low. This is surprising since the limited success that traditional approaches to support adherence have had and that technical interventions may easily be combined with other categories of interventions such as behavioural to address and potentially improve medication adherence. ${ }^{24}$
This is the first systematic literature review that aims to identify important design features of the PHR that may improve medication adherence in the adult population with long-term conditions.

\section{Objectives}

\section{Primary objective}

Identify the important design features of the PHR that may have improved medication adherence in the adult population with long-term conditions.

\section{Secondary objectives}

- Identify the PHR design features that may have improved medication adherence in the cases of:

- polypharmacy;

- specific long-term condition groups.

- Identify if there was a correlation between participants' demographic characteristics, their usage of PHRs and their medication adherence.

- Explore how implementation factors affected medication adherence.

\section{METHODS}

This systematic review is registered in the prospective register of systematic reviews (PROSPERO) and it follows the Preferred Reporting Items for Systematic Reviews and Meta-Analyses (PRISMA) guidelines. ${ }^{25}$ The PRISMA checklist is provided as an online additional file. The protocol for this study is published. ${ }^{6}$

\section{Study eligibility and selection}

The inclusion and exclusion criteria of this review are illustrated in table 1 and are detailed in the published protocol. ${ }^{6}$ As a result of the peer-review process, we have decided to make a change in our published protocol and to exclude systematic literature reviews and include only primary studies.

There have been no deviations from the protocol.

\section{Literature search}

Mendeley software ${ }^{26}$ was used as the bibliographic software. One author (EA) scanned the titles and abstracts and excluded studies that clearly did not meet the inclusion criteria. Full-text versions of the remaining articles were obtained and screened by the same author, using the criteria listed on table 1 . All the excluded studies were listed with at least one reason for exclusion. In case of uncertainty, the author PS was advised and there was a discussion until an agreement was made. The reference lists of the included studies were examined to identify additional relevant literature. A hand-search of JMIR Medical Informatics, BMC Medical Informatics and Decision Making and BMJ Open was conducted by EA to identify further literature, as they were the three most cited and most impactful journals in the search we had done.

The full search strategy can be found in online supplementary file. The search dates are from 1 January 2002 to 31 May 2018 and the search terms are: 
Table 1 Summary of the PICOS elements included and excluded in the systematic review

\section{Inclusion criteria}

\begin{tabular}{ll}
\hline Participants & Humans \\
Adults with at least one long-term condition \\
Patients who can self-administer their medication \\
Patients who are able to communicate freely and able \\
to self-manage their medication \\
Patients who are treated outside the hospital only
\end{tabular}

Intervention

Interventions of any type, intensity and frequency, which aim to investigate the effect of electronic PHRs in medication adherence, concordance, compliance or persistence

\begin{tabular}{|c|c|c|}
\hline Comparators & Non-PHR & $\begin{array}{l}\text { No comparison has been made with non-PHR or } \\
\text { with usual care }\end{array}$ \\
\hline Outcome & $\begin{array}{l}\text { Any outcome related to the effect of electronic PHRs } \\
\text { in medication adherence, concordance, compliance or } \\
\text { persistence. }\end{array}$ & $\mathrm{N} / \mathrm{A}$ \\
\hline Study design/type & $\begin{array}{l}\text { Primary studies published in the last } 15 \text { years, without } \\
\text { any geographical restriction }\end{array}$ & $\begin{array}{l}\text { Abstract-only reports without any references, } \\
\text { commercial studies, party political statements, } \\
\text { general discussion papers, magazine or } \\
\text { newspaper articles, withdrawn abstracts or } \\
\text { articles, protocols of reviews or literature reviews }\end{array}$ \\
\hline $\begin{array}{l}\text { Quality of the } \\
\text { studies }\end{array}$ & Studies with moderate or high quality & Studies with low and very low quality \\
\hline
\end{tabular}

PHR, personal health record.

(phr OR "personal health record" OR "patient portal”) AND adult* AND ("chronic disease" OR "chronic illness" OR "chronic condition" OR "long term disease" OR "long term illness" OR "long term condition") AND ("medication compliance" OR "medication adherence" OR "medication concordance" OR “medication persistence”)

\section{Data extraction and analysis}

EA reviewed and extracted all literature and the data in the predefined data extraction forms. ${ }^{6}$ The data extraction forms were designed by PS and EA to collect all the necessary data, based on the National Institute for Health and Care Excellence data extraction forms ${ }^{27}$ and the research questions. The completed data extraction forms, the initial and complete narrative analysis of the generated data were performed by EA and face-validated by $\mathrm{PS}^{6}$ who did not review the excluded literature.

\section{Quality assessment}

The quality of all included studies was assessed by the first author (EA) and 25\% of the included studies were assessed by the second author (PS). Cohen's Kappa (k) inter-rater reliability measure ${ }^{28}$ was calculated. The Joanna Briggs Institute (JBI) critical appraisal questionnaires were used to implement the GRADE approach $^{29}$ of quality assessment of the included studies. The GRADE approach is proposed by the Cochrane Collaboration ${ }^{30}$ and it favours the JBI critical appraisal tools. ${ }^{31}$

\section{Exclusion criteria}

Animals

Pregnant, cancer or terminally ill patients Adults with medically serious problems that are not classified as long-term conditions Patients who require assistance in taking their medication

Patients who are unable to communicate or unable to self-manage their medication Inpatients or patients who are living in care homes

N/A

The final quality scores per paper (table 2) were assigned based on five factors: risk of bias, ${ }^{32}$ inconsistency $^{33}$ indirectness, ${ }^{34}$ imprecision ${ }^{35}$ and publication bias. ${ }^{36}$ All the studies were graded based on their study type, for example, randomised controlled trials (RCTs) and systematic literature reviews were stated as high quality studies. Using the GRADE categories, we started from the highest possible score based on the study type, using the JBI questions to extract data per paper for the GRADE scoring, reducing the quality for each instance of the factors mentioned above.

\section{Patient and public involvement}

Patient and public involvement (PPI) has already been involved in the design of this research. On 22 June 2018, a PPI focus group with eight participants took place at the University of Portsmouth. The suggestions of this group are taken into consideration by the research team. The PPI group approved the focus of this review and provided guidelines for future research.

\section{RESULTS}

\section{Literature search results}

Figure 1 illustrates the literature search and selection method, presenting explanations for the exclusion of studies. Once duplicates were removed, a total of 


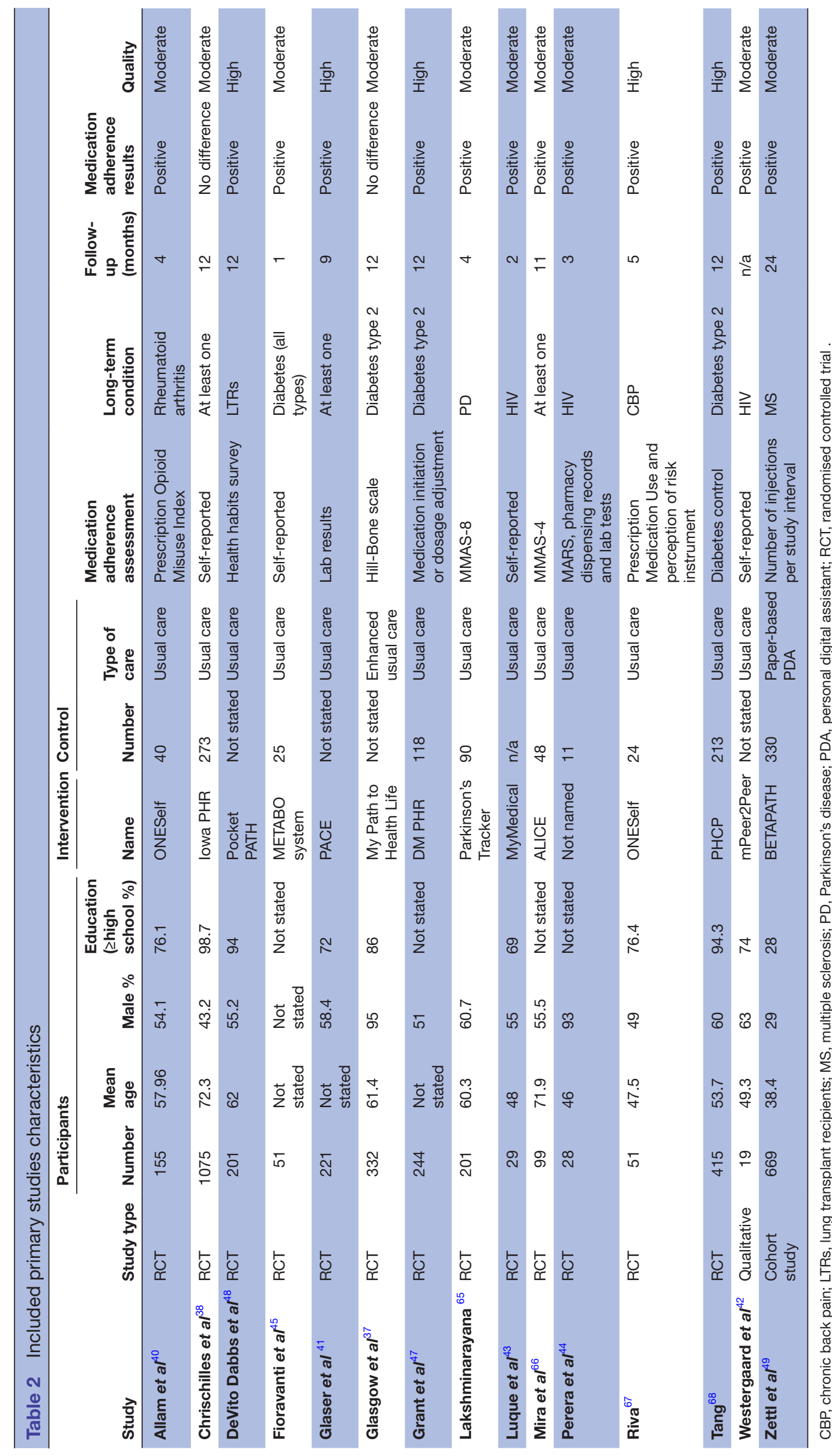




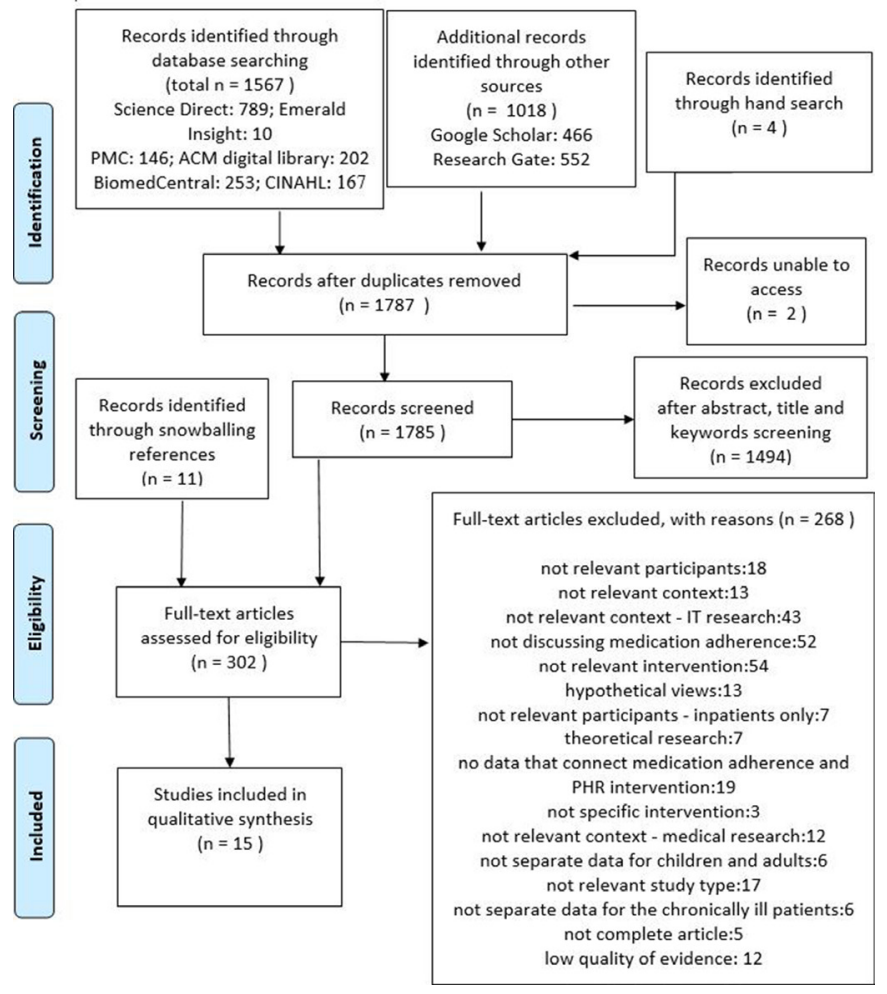

Figure 1 Preferred Reporting Items for Systematic Reviews and Meta-Analyses literature search and selection method diagram.
1787 original works were identified and 15 were finally included in the qualitative synthesis.

\section{Characteristics of included studies}

This review includes studies conducted in the USA $(n=7)$, Switzerland ( $\mathrm{n}=2)$, Canada $(\mathrm{n}=1)$, UK $(\mathrm{n}=1)$, Germany $(\mathrm{n}=1)$, Italy and Czech Republic $(\mathrm{n}=1)$, New Zealand $(\mathrm{n}=1)$ and Spain $(\mathrm{n}=1)$. Most studies had included participants who have at least one long-term condition $(n=9)$, diabetes $(n=4)$ and HIV $(n=3)$. Studies were published in $2017(\mathrm{n}=4), 2014(\mathrm{n}=4), 2016(\mathrm{n}=3), 2015 \quad(\mathrm{n}=2), 2013$ $(n=1)$ and $2008 \quad(n=1)$. The majority of the included studies $(n=13)$ reported a positive result in terms of the impact on medication adherence, with two studies found no difference. ${ }^{3738}$ No study identified negative results. Five out of 5 high quality studies and 8 out of 10 moderate quality studies found positive results with medication adherence. The main study characteristics are illustrated in table 2.

\section{Primary objective}

Table 3 presents the PHR design features that were identified in the literature and are the generated themes of this literature review. The definitions of the PHR design features were derived from commercial PHRs that are implementing them or the wider literature regarding PHRs and personal experience, since there are no

Table 3 PHR design features

\begin{tabular}{|c|c|c|c|}
\hline Feature & Definition & Positive & No difference \\
\hline Reminder & $\begin{array}{l}\text { Reminders to take medication or to reorder their prescriptions, agnostic to } \\
\text { the patient's chronic condition, demographics or any other external factor. }\end{array}$ & 11 & 1 \\
\hline Education & $\begin{array}{l}\text { Includes smaller features, like search bars and views, which can improve } \\
\text { the user's health literacy and understanding of their condition. }\end{array}$ & 3 & 2 \\
\hline Personalisation and tailoring & $\begin{array}{l}\text { The personalisation involves presenting a health message specific to } \\
\text { the individual patient's condition and demographic characteristics, } \\
\text { while tailoring involves developing a PHR based on the individual's } \\
\text { characteristics. } 69\end{array}$ & 8 & 1 \\
\hline Feedback and alerts & $\begin{array}{l}\text { Medical emergency alerts in the form of a press button alert or SMS, such } \\
\text { as the ones a user may find in the MyALERT PHR. }{ }^{70}\end{array}$ & 10 & 2 \\
\hline Gamification & $\begin{array}{l}\text { An umbrella term that includes the use of game design characteristics in } \\
\text { non-game contexts. }{ }^{71}\end{array}$ & 4 & 1 \\
\hline Medication management & $\begin{array}{l}\text { Includes all the features that a chronically ill patient may need to conform to } \\
\text { everyday life. }{ }^{22}\end{array}$ & 10 & 2 \\
\hline $\begin{array}{l}\text { Medical appointment } \\
\text { management }\end{array}$ & Medically related appointment tracking, re-scheduling and arrangement. ${ }^{73}$ & 2 & - \\
\hline Diary and self-monitoring & $\begin{array}{l}\text { A combination of features related to health or medication intake } \\
\text { monitoring. }{ }^{74}\end{array}$ & 8 & 2 \\
\hline $\begin{array}{l}\text { Health condition } \\
\text { management }\end{array}$ & $\begin{array}{l}\text { Includes all the lab and medical tests results and integration of existing } \\
\text { clinical data. }{ }^{69}\end{array}$ & 3 & 1 \\
\hline Set goals & Includes all the elements that are necessary for setting and managing goals. & 2 & 1 \\
\hline Patient's blog & Includes sharing in social media or blogging regarding health. ${ }^{40}$ & 1 & - \\
\hline Tethered & Are the PHRs which are connected with an EHR. ${ }^{75}$ & 2 & - \\
\hline
\end{tabular}

EHR, electronic health record; PHR, personal health record. 
International Standards Organization standards or universally accepted terminology.

All the PHR design features, which constitute the themes derived from our thematic analysis, ${ }^{39}$ are interlinked and overlapped. NVivo V.11 was used to cluster our codes potentially into more comprehensive themes. The initial themes were clustered together based on coding similarity, which means that if they were coding many of the same included studies, then they were clustered together.

- Reminder and medical appointment management (used in two studies).

- Tethered (used in two studies).

- Diary and self-monitoring (used in 10 studies).

- Feedback and alerts and health condition management (used in four studies).

- Medication management and patients blog (used in one studies).

- Personalisation and tailoring, gamification, education, set goals (used in 10 studies).

\section{Secondary objectives}

PHR design features that improve medication adherence in case of polypharmacy

Although multiple papers include polypharmacy or multimorbidity factors about their participants, they do not explore whether there is a correlation or an association between polypharmacy and the PHR usage or whether there is a medication adherence improvement or not. There is a trend that the more medications a person uses the less a reminder helps. This is not a conclusion directly supported by the data, but an argument emerging from multiple studies. ${ }^{38} 40-42$

\section{PHR design features that improve medication adherence for specific long-term condition groups}

This research identified a number of chronic conditions. However, diabetes and HIV can only be used to analyse the impact that the PHR design features are having in medication adherence, since the rest of the conditions are included in just one study.

\section{HIV}

Three studies have been included which discuss how the use of PHRs affect medication adherence of adults with HIV. ${ }^{42-44}$ All three studies produced positive results, having a very small (less than 50) number of participants who are also predominantly men and younger than 50 years old. Two studies are RCTs, ${ }^{43}{ }^{44}$ having follow-up duration of 2-3 months and the other one is a qualitative study ${ }^{42}$ without any follow-up. All three studies include the personalisation and tailoring design feature, two of the studies use reminders, one study uses feedback and alerts and health condition management and one other study uses diary and self-monitoring design feature.

\section{Diabetes}

Four studies have been included which discuss how the use of PHRs affect medication adherence of adults with diabetes. ${ }^{3745-47}$ All studies found positive results regarding medication adherence, apart from Glasgow et al. ${ }^{37}$ Three studies used diary and self-monitoring ${ }^{374} 46$ or personalisation and tailoring, ${ }^{37}{ }^{45-47}$ three studies used education and set goals ${ }^{374546}$ or gamification ${ }^{374547}$ and one ${ }^{46}$ study used feedback and alerts and health condition management PHR design features. The follow-up duration of studies was 12 months apart from Fioravanti $e t a l,{ }^{45}$ which was 1 month.

Association between participants' demographic characteristics, their usage of PHRs and their medication adherence

Data regarding patients' demographics and medication adherence were collected and analysed. Only $2^{43} 48$ of the 15 studies provide a detailed description of the participants' ethnicity. Two studies ${ }^{48}$ also provide data regarding the technology literacy of the participants and two other studies ${ }^{3743}$ provide data for the health literacy of the participants.

Twelve of the 15 studies provided age group details, which illustrate that the younger the participants, the more positive results regarding medication adherence were identified. This could be due to the presumed technological literacy of younger generations, however it was impossible to account for this confounding factor, since no data were supplied. Also the lower the number of participants in an experimental study (less than 251), the higher the impact of PHR in medication adherence. This could arguably illustrate a case of confirmation bias, meaning that the researcher stopped recruitment when the hypothesis was verified. It could also mean that some of these results are less accurate, due to lower number of participants, since there is uncertainty towards the validity of the results derived from RCTs with less than 100 participants. $^{50}$

\section{How implementation factors affect the outcomes and} heterogeneity

Heterogeneity has been found in the included studies regarding the study location, the included long-term conditions and the medication adherence measurement method. Although the included study types were also heterogenic (table 2), this does not seem to affect the general trend that the use of PHR has a positive impact in medication adherence. Heterogeneity has also been identified in participants' demographics and confounding factors (table 2).

Although expected otherwise, the duration of the follow-up in RCT studies does not seem to affect the outcomes, since the majority of the included RCTs produced positive results. There is however an indication that three out of four RCT studies that did not produce positive results had 12 months follow-up. This observation is in-line with the general idea of the field being new and there are no global standards that dictate the design of PHRs. Another interesting fact is that the studies that were either observational or literature reviews produced statistically more positive results than the RCTs. 


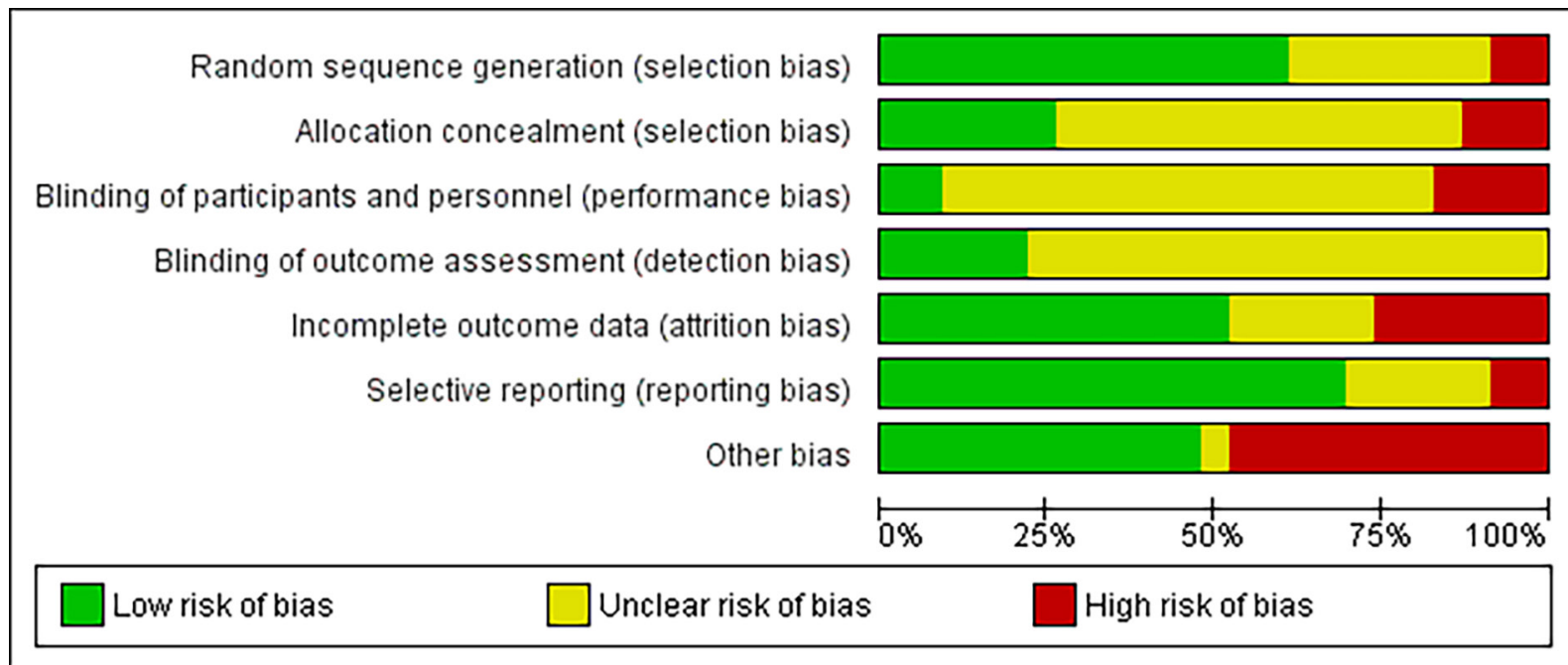

Figure 2 Overall risk of bias.

\section{Quality assessment}

The inter-rater reliability for quality assessment was calculated $\mathrm{k}=0.88$, which indicates high reliability of the quality assessment. Overall, 27 studies matched our inclusion criteria. Twelve of these studies were excluded due to low quality of evidence, 5e were graded as high quality of evidence and the remaining 10 were graded as moderate quality of evidence. The detailed quality assessment table based on the GRADE approach can be found in the online supplementary file 1 .

Figure 2 illustrates the overall risk of bias for this study. The details behind this diagram and the overall quality assessment are provided as additional file.

\section{DISCUSSION}

In this section, we reflect on the principal themes and overall conclusions from the literature review. The first impression after the initial data analysis was that too few studies actually mentioned the term PHR. In the majority of the cases, we had to critique the intervention of the inclusion criteria based on our PHR definition. This systematic review included 15 primary studies of multiple study types and identified 12 different PHR design features. Although 12 different PHR design features were identified, there are no specific guidelines that can be derived from the results. Overall, $87 \%(n=13)$ of the included studies found that the use of a PHR has increased medication adherence. Based on the number of studies that identified positive results, the fact that 8 out of the 10 high quality studies identified positive results and that no study indicated a negative effect on medication adherence, we conclude that there is a reasonable indication that PHRs can have an overall positive effect on medication adherence. However, due to the high heterogeneity in medication adherence measurements, reporting styles and study types, we were unable to group and quantify the results in study level. It is of course possible that the absence of negative results is due to publication bias.
Reminders, feedback and alerts and medication management are the most commonly used PHR design feature, since all studies include at least one of the three features. These findings are also consistent with the existing literature, which identifies reminders, and medication management as the most commonly used features that assist patients with their medication intake. ${ }^{51-55}$ The design features with the most positive effect for patients' medication adherence were patient's blog, tethered and medical appointment management. This could be arguable since these features are also the least used, since the medication appointment management has the most positive results in the relevant literature. ${ }^{52-58}$ The generated clusters paint a similar picture. The cluster that produces the best results is personalisation and tailoring, gamification, education and set goals (92\% of studies in this cluster produce positive results) and reminder and medical appointment management $(80 \%$ of studies in this cluster produce positive results).

Two groups of patients have been identified in this literature review for further analysis to find which of the PHR design features work best for them. The most positive results for diabetic participants were identified by three studies ${ }^{45-47}$ that used PHRs. Furthermore, for participants with HIV, three studies were identified producing positive results ${ }^{42-44}$ that used at least personalisation and tailoring design feature and reminders. Mixed associations were found between patients demographics, their PHR usage and medication adherence. Taking into account the median age confounding factor of the participants, it is apparent that the younger the participants, the more positive are the results. This is also supported in the general academic literature that suggests that technological literacy levels are higher in younger adult population. ${ }^{59} 60$

Furthermore, the less participants a study included the more positive the results appeared to be. This in fact may cause problems in the quality of evidence for the 
included studies and this literature review; hence our suggestion is to interpret the results with caution. There is a proven link between the sample size of an RCT and the statistical significance of its result. ${ }^{61}$ According to Faber and Fonseca, ${ }^{62}$ the small sample size might increase the chance of false-positive and the study might not reach to an evident conclusion.

The 'other' bias category has the highest risk of bias of all the assessed risks, which is in line with the key findings of this and other related studies, ${ }^{63}$ resulting in a probable overestimation of PHR effect on medication adherence and potentially echoing a form of recall bias. ${ }^{64}$ Although some degree of bias is considered unavoidable, the high risk of bias identified in the included studies and the generally moderate quality of evidence presented in them also reflect the uncertainty on this field and the need for further research on both PHRs and medication adherence. A PHR design feature that is not mentioned in the included literature is security. This might be happening due to the inclusion/exclusion criteria of this systematic review, since the security of the data does not seem to affect patients' medication adherence. Another limitation is that we included English papers published in the last 15 years, due to time and cost constraints. Screening of the studies and data collection were conducted by EA and only face-validated by PS, therefore this is also a significant limitation. Furthermore, the small number of participants in the studies is commonly known to overestimate the effect of an intervention, the fact which is noticeable in the included studies.

Acknowledgements The authors would like to acknowledge Dr Nicola Barnes and Dr Penny Ross, for providing academic insight and support. The authors would also like to acknowledge the participants of the PPI group for their insightful feedback and time.

Contributors EA drafted the manuscript, and he is the guarantor of the review. PS revised the manuscript multiple times for methodological and intellectual content. $\mathrm{HH}$ revised the manuscript twice for methodological, conceptual and intellectual content from a pharmaceuticals' perspective. AG also revised the manuscript for methodological, conceptual and intellectual content and also contributed in the abstract design. The final version of the manuscript was approved by all the latter three authors.

Funding The authors have not declared a specific grant for this research from any funding agency in the public, commercial or not-for-profit sectors.

Competing interests None declared.

Patient consent for publication Not required.

Provenance and peer review Not commissioned; externally peer reviewed.

Data availability statement All data relevant to the study are included in the article or uploaded as supplementary information.

Open access This is an open access article distributed in accordance with the Creative Commons Attribution Non Commercial (CC BY-NC 4.0) license, which permits others to distribute, remix, adapt, build upon this work non-commercially, and license their derivative works on different terms, provided the original work is properly cited, appropriate credit is given, any changes made indicated, and the use is non-commercial. See: http://creativecommons.org/licenses/by-nc/4.0/.

\section{REFERENCES}

1. Deloitte. 2018 global health care outlook: the evolution of smart health care, 2018. Available: https://www2.deloitte.com/content/
dam/Deloitte/global/Documents/Life-Sciences-Health-Care/gx-Ishchc-outlook-2018.pdf [Accessed 22 Nov 2018].

2. National Information Board, Department of Health. Personalised health and care 2020: a framework for action, 2014. Available: https://www.gov.uk/government/publications/personalised-healthand-care-2020/using-data-and-technology-to-transform-outcomesfor-patients-and-citizens [Accessed 11 Feb 2018].

3. NHS. The NHS long term plan, 2019. Available: www.longtermplan. nhs.uk [Accessed 2 May 2019].

4. Office for National Statistics. Internet users in the UK: Statistical Bulletin, 2016. Available: https://www.ons.gov.uk/businessindu stryandtrade/itandinternetindustry/bulletins/internetusers/2016 [Accessed 25 Sep 2018].

5. Deloitte. 2017 global health care sector outlook, 2017. Available: https://www2.deloitte.com/content/dam/Deloitte/global/Documents/ Life-Sciences-Health-Care/gx-Ishc-2015-health-care-outlookinfographic.pdf [Accessed 10 Nov 2018].

6. Andrikopoulou E, Scott PJ, Herrera H. Important design features of personal health records to improve medication adherence for patients with long-term conditions: protocol for a systematic literature review. JMIR Res Protoc 2018;7:e159.

7. Price M, Bellwood P, Kitson N, et al. Conditions potentially sensitive to a personal health record (PHR) intervention, a systematic review. BMC Med Inform Decis Mak 2015;15:32.

8. Institute of Medicine. Health it and patient safety. Washington, D.C: National Academies Press, 2012.

9. Moorhead P, Zavala A, Kim Y, et al. Efficacy and safety of a medication dose reminder feature in a digital health offering with the use of sensor-enabled medicines. J Am Pharm Assoc 2017;57:155-61.

10. De Geest S, Sabaté E. Adherence to long-term therapies: evidence for action. Eur $J$ Cardiovasc Nurs 2003;2.

11. Rapoff MA. Adherence to pediatric medical regimens. Boston, MA: Springer US, 2010

12. McDonald HP, Garg AX, Haynes RB. Interventions to enhance patient adherence to medication prescriptions. JAMA 2002;288:2868-79.

13. Who. Defining adherence, 2003. Available: www.who.int/chp/ knowledge/publications/adherence_report/en/ [Accessed 25 Oct 2017].

14. Clyne B, Bradley MC, Hughes C, et al. Electronic prescribing and other forms of technology to reduce inappropriate medication use and polypharmacy in older people: a review of current evidence. Clin Geriatr Med 2012;28:301-22.

15. Kvarnström K, Airaksinen M, Liira H. Barriers and facilitators to medication adherence: a qualitative study with general practitioners. BMJ Open 2018;8:e015332.

16. Duerden M, Avery T, Payne R. Polypharmacy and medicines optimisation making it safe and sound, 2013. Available: http:// citeseerx.ist.psu.edu/viewdoc/download?doi=10.1.1.734.5288\&rep= rep1\&type=pdf [Accessed 25 Nov 2017].

17. DH/Long Term Conditions. Long term conditions compendium of information: third edition, 2012. Available: https://assets.publishing. service.gov.uk/government/uploads/system/uploads/attachment_ data/file/216528/dh_134486.pdf [Accessed 1 Mar 2019].

18. Royal Pharmaceutical Society. Medicines adherence, 2015. Available: https://www.rpharms.com/resources/quick-referenceguides/medicines-adherence [Accessed 24 Oct 2017].

19. Poole ES. $\mathrm{HCl}$ and mobile health interventions. Trans/ Behav Med 2013;3:402-5.

20. Poole ES. $\mathrm{HCl}$ and mobile health interventions: how humancomputer interaction can contribute to successful mobile health interventions. Transl Behav Med 2013;3:402-5.

21. NHS Digital. Standards for commissioning or developing personal health records, 2018. Available: https://digital.nhs.uk/services/ personal-health-records-adoption-service/personal-healthrecords-adoption-toolkit/developing-a-personal-health-record/ standards-for-developing-personal-health-records [Accessed 1 Feb 2019].

22. Chisholm MA. Enhancing transplant patients' adherence to medication therapy. Clin Transplant 2002;16:30-8.

23. Nunes V, Neilson J, O'Flynn N, et al. Clinical guidelines and evidence review for medicines adherence: involving patients in decisions about prescribed medicines and supporting adherence full guideline. Natl Collab Cent Prim Care 2009:1-364.

24. Chisholm-Burns MA, Spivey CA. Pharmacoadherence: a new term for a significant problem. Am J Health Syst Pharm 2008;65:661-7.

25. Knobloch K, Yoon U, Vogt PM. Preferred reporting items for systematic reviews and meta-analyses (PRISMA) statement and publication bias. J Craniomaxillofac Surg 2011;39:91-2.

26. Elsevier. Mendeley, 2019. Available: https://www.mendeley.com [Accessed 28 Jun 2019]. 
27. The British Psychological Society. Data extraction forms for qualitative studies. In: A NICE-SCIE guideline on supporting people with dementia and their carers in health and social care. The British Psychological Society and Gaskell, 2006: 66-8.

28. Friedman CP, Wyatt J. Evaluation methods in biomedical informatics. New York: Springer-Verlag, 2006

29. Joanna Briggs Institute. Critical appraisal tools, 2006. Available: http://joannabriggs.org/research/critical-appraisal-tools.html [Accessed 4 Sep 2017].

30. Higgins J, Green S. Cochrane handbook for systematic reviews of interventions. version 5. The Cochrane collaboration, 2011. Available: www.handbook.cochrane.org [Accessed 13 Nov 2017].

31. Cochrane Training. Grade approach, 2018. Available: https://training cochrane.org/grade-approach [Accessed 4 Oct 2018].

32. Guyatt GH, Oxman AD, Vist G, et al. GRADE guidelines: 4. Rating the quality of evidence-study limitations (risk of bias). J Clin Epidemiol 2011;64:407-15.

33. Guyatt GH, Oxman AD, Kunz R, et al. Grade guidelines: 7 . Rating the quality of evidence-inconsistency. $J$ Clin Epidemiol 2011;64:1294-302.

34. Guyatt GH, Oxman AD, Kunz R, et al. GRADE guidelines: 8. Rating the quality of evidence-indirectness. J Clin Epidemiol 2011;64:1303-10.

35. Guyatt GH, Oxman AD, Kunz R, et al. GRADE guidelines 6 . Rating the quality of evidence--imprecision. J Clin Epidemiol 2011;64:1283-93.

36. Guyatt GH, Oxman AD, Montori V, et al. GRADE guidelines: 5 . Rating the quality of evidence-publication bias. J Clin Epidemiol 2011;64:1277-82.

37. Glasgow RE, Kurz D, King D, et al. Twelve-month outcomes of an Internet-based diabetes self-management support program. Patient Educ Couns 2012;87:81-92.

38. Chrischilles EA, Hourcade JP, Doucette W, et al. Personal health records: a randomized trial of effects on elder medication safety. $J$ Am Med Inform Assoc 2014;21:679-86.

39. Braun V, Clarke V. Using thematic analysis in psychology. Qual Res Psychol 2006;3:77-101.

40. Allam A, Kostova Z, Nakamoto K, et al. The effect of social support features and gamification on a web-based intervention for rheumatoid arthritis patients: randomized controlled trial. J Med Internet Res 2015;17:e14

41. Glaser E, Richard C, Lussier M-T. The impact of a patient web communication intervention on reaching treatment suggested guidelines for chronic diseases: a randomized controlled trial. Patient Educ Couns 2017:100:2062-70.

42. Westergaard RP, Genz A, Panico K, et al. Acceptability of a mobile health intervention to enhance HIV care coordination for patients with substance use disorders. Addict Sci Clin Pract 2017;12.

43. Luque AE, Corales R, Fowler RJ, et al. Bridging the digital divide in HIV care: a pilot study of an iPod personal health record. $J$ Int Assoc Provid AIDS Care 2013;12:117-21.

44. Perera Al, Thomas MG, Moore JO, et al. Effect of a smartphone application incorporating personalized health-related imagery on adherence to antiretroviral therapy: a randomized clinical trial. AIDS Patient Care STDS 2014;28:579-86.

45. Fioravanti A, Fico G, Salvi D, et al. Automatic messaging for improving patients engagement in diabetes management: an exploratory study. Med Biol Eng Comput 2015;53:1285-94.

46. Tang PC, Ash JS, Bates DW, et al. Personal health records: definitions, benefits, and strategies for overcoming barriers to adoption. J Am Med Inform Assoc 2006;13:121-6.

47. Grant RW, Wald JS, Schnipper JL, et al. Practice-linked online personal health records for type 2 diabetes mellitus: a randomized controlled trial. Arch Intern Med 2008;168:1776-82.

48. DeVito Dabbs A, Song MK, Myers BA, et al. A randomized controlled trial of a mobile health intervention to promote self-management after lung transplantation. Am J Transplant 2016;16:2172-80.

49. Zettl UK, Bauer-Steinhusen U, Glaser T, et al. Adherence to longterm interferon beta- $1 \mathrm{~b}$ injection therapy in patients with multiple sclerosis using an electronic diary. Adv Ther 2016;33:834-47.

50. Spieth PM, Kubasch AS, Penzlin Al, et al. Randomized controlled trials - a matter of design. Neuropsychiatr Dis Treat 2016;12:1341-9.

51. Hayes TL, Cobbinah K, Dishongh T, et al. A study of medicationtaking and unobtrusive, intelligent reminding. Telemed J E Health 2009;15:770-6.

52. Mistry N, Keepanasseril A, Wilczynski NL, et al. Technology-mediated interventions for enhancing medication adherence. J Am Med Inform Assoc 2015;22:e177-93.
53. Harrison S, Stadler M, Ismail K, et al. Are patients with diabetes mellitus satisfied with technologies used to assist with diabetes management and coping?: a structured review. Diabetes Technol Ther 2014:16:771-83.

54. McLean G, Murray E, Band R, et al. Interactive digital interventions to promote self-management in adults with asthma: systematic review and meta-analysis. BMC Pulm Med 2016;16:83.

55. Morton K, Dennison L, May C, et al. Using digital interventions for self-management of chronic physical health conditions: a metaethnography review of published studies. Patient Educ Couns 2017;100:616-35.

56. Dorr D, Bonner LM, Cohen AN, et al. Informatics systems to promote improved care for chronic illness: a literature review. J Am Med Inform Assoc 2007;14:156-63.

57. Park LG, Howie-Esquivel J, Dracup K. A quantitative systematic review of the efficacy of mobile phone interventions to improve medication adherence. J Adv Nurs 2014:70:1932-53.

58. Stephani V, Opoku D, Quentin W. A systematic review of randomized controlled trials of mHealth interventions against non-communicable diseases in developing countries. BMC Public Health 2016;16:572.

59. Bastion S. The development of a valid and reliable measure of technological literacy for adults, 2014. Available: https:// kuscholarworks.ku.edu/handle/1808/18642

60. Sharp LA. Collaborative digital literacy practices among adult learners: levels of confidence and perceptions of importance. Int $J$ Instruction 2018;11:153-66.

61. Walsh M, Srinathan SK, McAuley DF, et al. The statistical significance of randomized controlled trial results is frequently fragile: a case for a fragility index. J Clin Epidemiol 2014;67:622-8.

62. Faber J, Fonseca LM. How sample size influences research outcomes. Dental Press J Orthod 2014;19:27-9.

63. Showell C. Barriers to the use of personal health records by patients: a structured review. PeerJ 2017:5:e3268.

64. Tsai K-T, Chen J-H, Wen C-J, et al. Medication adherence among geriatric outpatients prescribed multiple medications. Am J Geriatr Pharmacother 2012;10:61-8.

65. Lakshminarayana $\mathrm{R}$, Wang $\mathrm{D}$, Burn $\mathrm{D}$, et al. Using a smartphonebased self-management platform to support medication adherence and clinical consultation in Parkinson's disease. NPJ Parkinsons Dis 2017;3.

66. Mira JJ, Navarro I, Botella F, et al. A Spanish pillbox APP for elderly patients taking multiple medications: randomized controlled trial. $J$ Med Internet Res 2014;16:e99.

67. Riva S, Camerini A-L, Allam A, et al. Interactive sections of an Internet-based intervention increase empowerment of chronic back pain patients: randomized controlled trial. J Med Internet Res 2014;16:e180.

68. Tang PC, Overhage JM, Chan AS, et al. Online disease management of diabetes: engaging and motivating patients online with enhanced resources-diabetes (EMPOWER-D), a randomized controlled trial. $J$ Am Med Inform Assoc 2013;20:526-34.

69. Househ MS, Borycki EM, Rohrer WM, et al. Developing a framework for meaningful use of personal health records (PHRs). Heal Policy Technol 2014;3:272-80.

70. ALERT Life Sciences Computing. MyALERT® - Personal Health Record, 2010. Available: http://www.alert-online.com/myalert [Accessed 26 Oct 2017].

71. Deterding S, Sicart M, Nacke L, et al. Gamification. using gamedesign elements in non-gaming contexts. In: Proceedings of the 2011 annual conference extended abstracts on Human factors in computing systems - CHI EA '11. New York, New York, USA: ACM Press, 2011: 2425.

72. Dalgaard L, Grönvall E, Verdezoto N. Accounting for Medication Particularities: Designing for Everyday Medication Management In: Proceedings of the ICTS for improving patients rehabilitation research techniques. ICST, Brussels, Belgium, Belgium: IEEE, 2013: 137-44.

73. Wagner PJ, Dias J, Howard S, et al. Personal health records and hypertension control: a randomized trial. J Am Med Inform Assoc 2012;19:626-34

74. Zettl UK, Bauer-Steinhusen U, Glaser T, et al. Evaluation of an electronic diary for improvement of adherence to interferon beta- $1 \mathrm{~b}$ in patients with multiple sclerosis: design and baseline results of an observational cohort study. BMC Neurol 2013;13:117.

75. Vydra TP, Cuaresma E, Kretovics M, et al. Diffusion and use of tethered personal health records in primary care. Perspect Heal Inf Manag 2015;12. 